\title{
Effects of musical ear training on lexical tone perception
}

\author{
Evan D. Bradley \& Janet G. Van Hell*
}

\begin{abstract}
The effect of short term musical experience on lexical tone perception was examined by administering four hours of daily musical ear training to non-tone language speakers. After training, participants showed some improvement in a tone labeling task, but not a tone discrimination task; however, this improvement did not differ reliably from controls indicating that short-term musical training is thus far not able to replicate language effects observed among lifelong musicians, but some linguistic differences between musicians and nonmusicians may likely be due to experience, rather than individual differences or other factors.
\end{abstract}

Keywords. lexical tone; Mandarin; music; perception; melody; learning.

1. Introduction. Musicians perceive pitch differently from others, which is reflected in their perception, production, and learning of lexical tones (Bradley, 2013). Musicians also possess more robust phonetic pitch representations, which are prerequisite for acquiring tonal categories and words (Wong \& Perrachione, 2007). Musicianship has therefore been argued to change linguistic pitch perception, but cross-sectional evidence remains circumstantial, because musiclanguage studies typically compare musicians with many years of training to those with little to no formal training. It remains poorly established how what music skill level or degree of training is required to see differences in linguistic pitch perception, but differences likely arise from a combination of explicit training, implicit learning, and individual aptitudes and personalities (Corrigall, Schellenberg, \& Misura, 2013). Neural differences associated with musical experience suggest that musical training could be harnessed for non-musical gains (Kraus \& Chandrasekaran, 2010; Patel, 2011).

To determine to what degree explicit perceptual musical training shapes linguistic perception (and whether such shaping can still occur in adulthood), we administered computerized musical ear training to non-tone language speakers who were not musicians in order to determine:

1. Whether music-induced changes in lexical tone perception experience be observed among adult non-tone language speakers.

2. What musical skill level or degree of training is sufficient to produce changes in lexical tone perception.

Because musicians use ear training to increase sensitivity to musical pitch, if nonmusicians improve their pitch perception, we hypothesized that they will also benefit from the same linguistic "side effects," namely:

1. Improved perception of unfamiliar lexical tones;

2. Improvement primarily in phonetic (vs. phonological) tasks.

* This project was supported by a grant from the Penn State Social Science Research Institute and Penn State Brandywine. The authors also thank Ingemarie Donker for assistance. Authors: Evan Bradley, The Pennsylvania State University (Brandywine) (evan.d.bradley@psu.edu) \& Janet G. Van Hell, The Center for Language Science, The Pennsylvania State University (University Park) and Radboud University, Nijmegen, the Netherlands (jgv3@psu.edu). 
2. Procedure. Participants were 32 non-tone language speakers (L1 English $=24)$ with musical backgrounds ranging from no musical participation to moderate amateur participation-however, none were professional musicians, and none had studied music theory or aural skills $(\mathrm{m}=3.9$ years instrument study).

Participants completed 4 hours of computerized aural skills (ear) training in daily one-hour sessions over one week, as follows.

- Day 1: Lexical Tone Pre-tests +30 minutes Ear Training

- Days 2-4: 1 hour Ear Training

- Day 5: 30-minute Ear Training + Lexical Tone Post-tests

2.1. EAR-TRAINING. Ear training is perceptual training to identify elements of music (e.g., scales, rhythms) through active listening and feedback. Training was administered through software (EarMaster) which controls training parameters and tracks progress.

Participants were randomly assigned to two training conditions:

1. Interval Training $(\mathrm{n}=16)$, in which participants heard synthesized piano intervals (melodic note pairs defined by the distance/ratio between their pitches). During training, participants were asked to compare (say which pitch distance was larger) and name (select the appropriate label) intervals. The difficulty increased as training progressed through the addition of more similar interval types. Interval training was expected to boost lexical tone performance due to its basis in pitch.

2. Rhythmic Training $(n=16)$, in which participants repeated computer generated drum rhythms by tapping on the space bar. The difficulty increased as training progressed through the addition of longer and more complex rhythms. Unlike the interval training, this training involved production, as well as perception, but it was the only rhythmic task available in EarMaster which does not require the participant to read musical notation. Rhythmic training was not expected to affect lexical tone perception, because it does not involve pitch.

Figure 1. Intervals used during

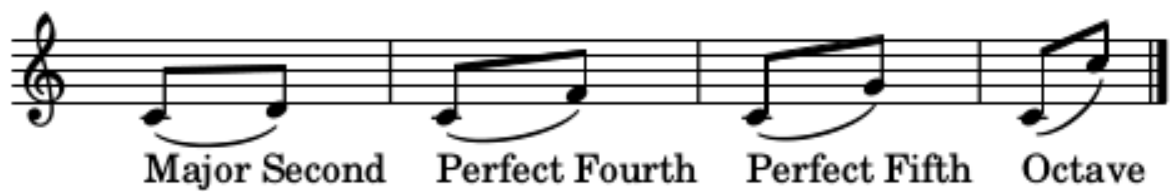
ear training.

2.2. LEXICAL TONE TESTS. Before and after training, tone perception was assessed through two tests based on Mandarin lexical tones. Stimuli both tasks consisted of the syllables [ma], [ku], and [di] spoken with each of the four Mandarin lexical tones by two native speakers (1 female, 1 male) in citation form. Presented with PsychoPy software, and responses were collected via keyboard.

1. Tone Labeling Task: participants matched a Mandarin word with a visual symbol depicting its pitch pattern $(\rightarrow \searrow \nearrow U)$. The test contained eight blocks of 24 trials (192 total); four blocks had a single speaker (low-variability) and four had two speakers (highvariability). 
2. Tone Comparison Task: Participants decided whether two words are the "same" or "different." Both words in a trial had the same syllable, and their tone differed on twothirds of trials ("same" trials had two different tokens of the same word). The test contained nine blocks of 20 trials (180 total); six blocks had a single speaker (lowvariability) and three had two speakers per trial (high-variability).

3. Results \& Discussion. Results were analyzed using separate analyses of variance for the two tone tests, with accuracy as the dependent variable, and session and group as independent variables. Neither group improved their performance in Tone Comparison. For Tone Labeling (see Figure 2), there was a marginally significant main effect of session, $\mathrm{F}(1,30)=3.70, p=$ 0.059 ; both the Interval and Rhythm groups improved slightly from pre-test to post-test (7.1\% vs. $6.1 \%$, respectively), although the size of this improvement did not differ significantly between the groups. Unexpectedly, there was a main effect of group, with the Interval Training group performing better than the Rhythmic Training group at both pre-test and post-test, $\mathrm{F}(1,30)$ $=6.08, p=0.017$; this was surprising, given that participants were randomly assigned to training conditions, and could possibly confound the results by flattening any expected greater gains by the interval group if they were already outperforming the control group.

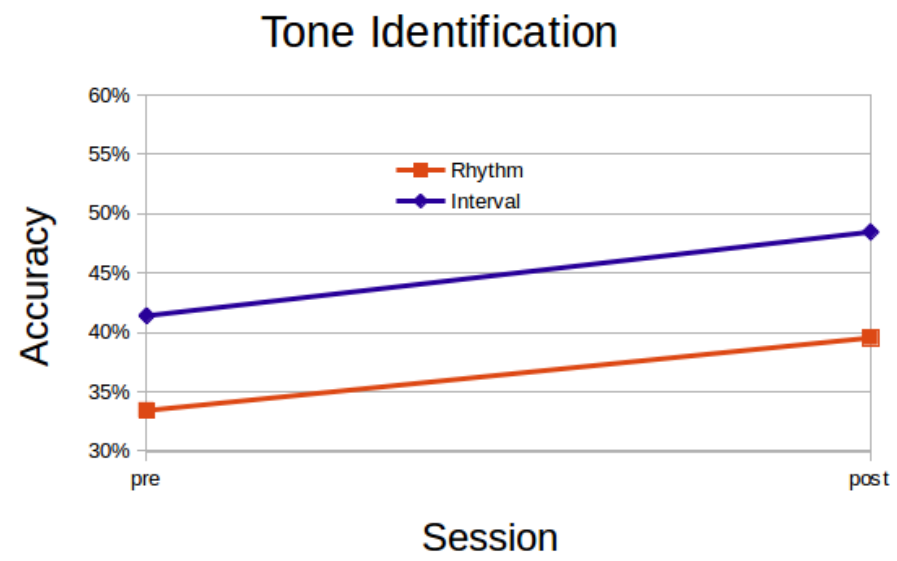

Figure 2. Changes in Mandarin Tone Identification after ear training.

Post-hoc, Bonferroni-adjusted t-tests were used to compare performance in low- and highvariability blocks of the Tone Labeling Task (see Figure 3). In low-variability (1-speaker) blocks, the Interval group improved in Labeling more than the Rhythm group ( $8.0 \%$ vs $5.5 \%)$, but this was not significant $(t(29)=-0.57, p=0.29)$. Labeling improvement in the high-variability (2speaker) blocks was similar for the Interval and Rhythm groups (6.1\% vs. $6.7 \%)$. 


\section{Tone Identification by Variability}

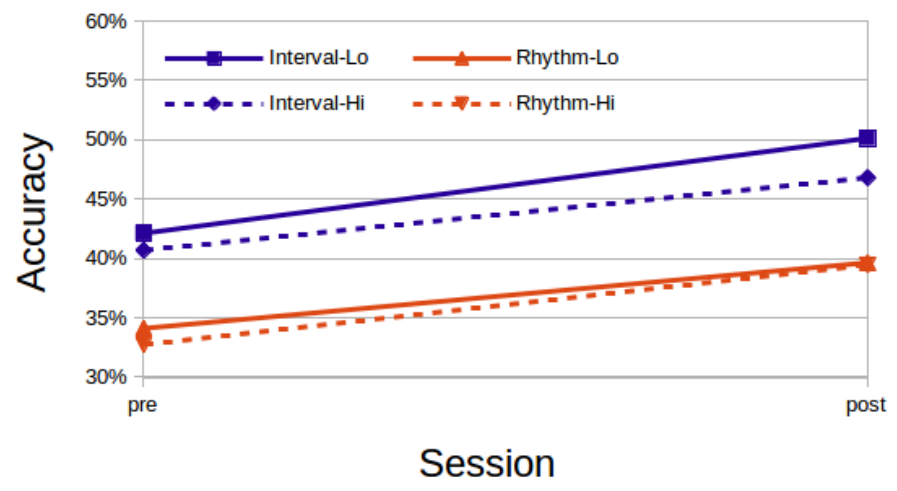

Figure 3. Changes in high- and low-variability Mandarin Tone Identification after ear training.

4. Conclusions. Four hours of melodic interval ear training does not seem sufficient to improve tone perception relative to rhythmic training. There is some evidence that those achieving more in interval training improved more in tone, but additional data are needed. If interval training affects tone perception, it affects Tone Labeling more than it does Tone Comparison (category matching). This would be consistent with progression along a phonetic-phonological continuity (Wong \& Perrachione, 2007), if training affects phonetic (pitch shape, in its low-variability, more phonetic condition) but not phonological (category decision) tasks. Further studies with increased training with additional contexts (e.g., pitch production) are necessary.

\section{References}

Bradley, E. D. (2013). Pitch perception in lexical tone and melody. Reviews of Research in Human Learning and Music, 1. doi: 10.6022/journal.rrhlm.2013002

Corrigall, K. A., Schellenberg, E. G., \& Misura, N. M. (2013). Music training, cognition, and personality. Frontiers in Psychology, 4(222). doi: 10.3389/fpsyg.2013.00222

Kraus, N., \& Chandrasekaran, B. (2010). Music training for the development of auditory skills. Nature Reviews Neuroscience, 11(8), 599-605. doi: 10.1038/nrn2882

Patel, A. D. (2011). Why would musical training benefit the neural encoding of speech? the OPERA hypothesis. Frontiers in Psychology, 2(142). doi: 10.3389/fpsyg.2011.00142

Wong, P. C., \& Perrachione, T. K. (2007). Learning pitch patterns in lexical identification by native English-speaking adults. Applied Psycholinguistics, 28(4), 565-585. doi: $10.1017 / \mathrm{S} 0142716407070312$ 